\title{
Lessons learned from SCUBA-2 for future cryogenic instrumentation in space
}

\author{
Adam L. Woodcraft \\ SUPA, Institute for Astronomy, Edinburgh University, Blackford Hill, Edinburgh EH9 3HJ, UK; \\ UK Astronomy Technology Centre, Blackford Hill, Edinburgh EH9 3HJ, UK
}

\begin{abstract}
Various planned space astronomy missions such as SPICA, SAFIR, Constellation-X and XEUS will require detectors operating at ultra-low temperatures. Our current relevant experience in space is limited, and future instruments are in any case likely to have more demanding requirements. We must therefore take advantage of experience on the ground. The SCUBA-2 ground-based instrument is probably the largest and (thermally) most complex astronomical instrument ever built to operate at such low temperatures. The thermal design has been very successful, and I discuss techniques we have developed and lessons we have learned that will be applicable to future space missions.
\end{abstract}

Keywords: Sub-mm, instrumentation, SCUBA-2, ULT, space, thermal design

\section{INTRODUCTION}

Building successful scientific instruments which operate at ultra-low temperatures is a challenging occupation. The same is true for instruments designed for operation in space. Achieving both at once is even harder. Despite this, there are many planned missions which will require this challenge to be met. Unfortunately, our current experience in this area is limited to a small number of instruments, as can be seen from Fig. 1. Of the six instruments shown which have already been constructed, three have yet to be launched, and of the three that have been launched, two were unsuccessful. Nevertheless, as can be seen from Fig. 1, a large number of proposed missions will require ultra-low temperature (ULT) operation.

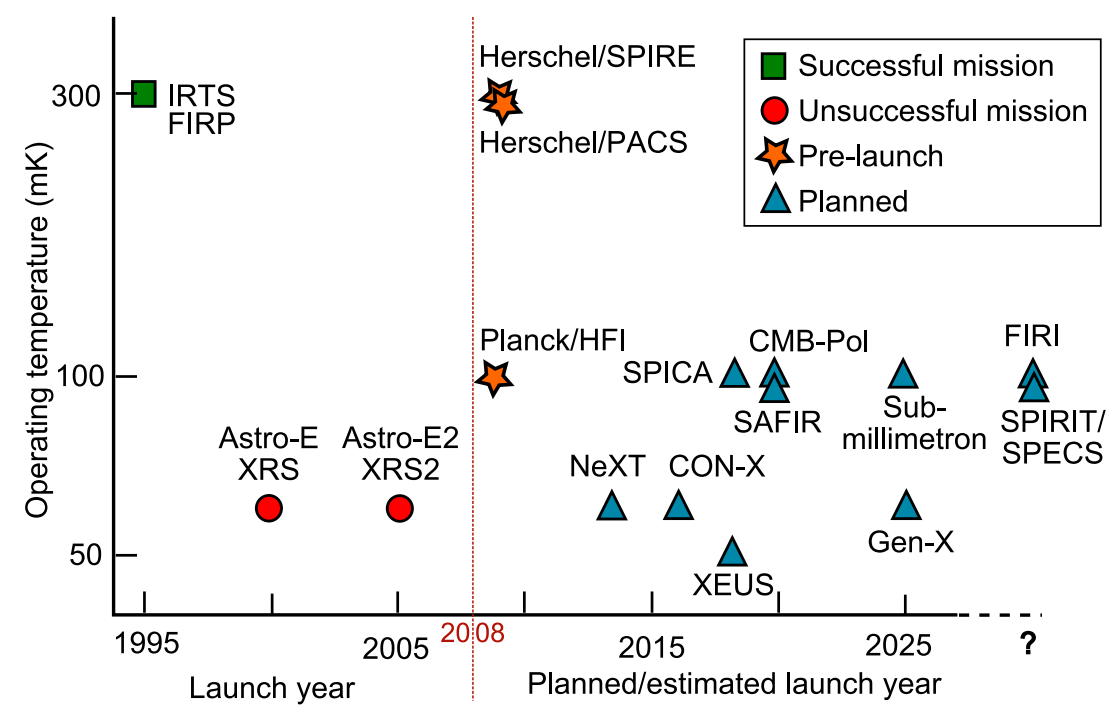

Figure 1. Existing and planned ultra-low temperature (below $1 \mathrm{~K}$ ) space cryogenic missions, with actual or planned launch dates. Instrument as well as mission names are shown for instruments which have already been constructed. Missions and possible launch dates were taken partially from Ref. 1 .

Further author information:E-mail: adam.woodcraft@physics.org, Telephone: +44 8707651873

Copyright 2008 Society of Photo-Optical Instrumentation Engineers. This paper was published in Proc. SPIE 7010 and is made available as an electronic reprint with permission of SPIE. One print or electronic copy may be made for personal use only. Systematic or multiple reproduction, distribution to multiple locations via electronic or other means, duplication of any material in this paper for a fee or for commercial purposes, or modification of the content of the paper are prohibited. 
The planned missions vary from some which are already in the study phase to others which are mere aspirations, and it is, of course, unlikely that all the proposed missions shown will eventually fly. However, barring a breakthrough in technology, future far infrared/sub-millimetre and X-ray space telescopes will employ ultra-low temperature detectors. ULT operation in space therefore has a likely future, and in any case some missions are already at the stage where the thermal design must be considered in detail, even if ultimately they are not selected.

The limited experience is not quite as bad as it might appear. The lack of success of the XRS instruments was not caused by any failure in the ULT systems. Indeed XRS-2 operated for long enough to demonstrate that the ultra-low temperature design performed well, before it was let down by the loss of the main helium bath which provided pre-cooling. Furthermore, the design and construction of the existing six instruments, along with studies on other missions which never flew, have already generated a considerable body of experience. Another advantage is that a large part of an ULT cooling chain consists of the systems that cool to temperatures of a few Kelvin; experience at these temperatures in space is (relatively) extensive, as can be seen from Fig. 2. However, the various advantages are tempered by the fact that future missions are likely to have significantly more demanding requirements than have been already encountered. In order to meet these requirements, we must look not only to our experience with space cryogenics, but to ULT instruments which have been built on the ground, both within the field of astronomy and outside.

The SCUBA-2 instrument, ${ }^{5}$ currently being commissioned on the James Clerk Maxwell Telescope in Hawaii, is probably the largest and (thermally) most complex astronomical instrument ever built to operate at such low temperatures. ${ }^{6}$ Future space missions will have to achieve similar levels of complexity but with the mass, power, volume and other constraints of an instrument in space. Even with the less stringent requirements of a ground-based instrument, the SCUBA-2 design required various novel solutions. The thermal design was nevertheless very successful. In this paper I discuss some of the techniques used and developed specifically for the instrument, as well as some general lessons (both what to do and what not to do) learned during the design of the instrument.

A final point to note is that SCUBA-2 employs mechanical coolers rather than a helium bath to provide the cryogenic environment. Future space instruments are also likely to use mechanical coolers due to the improved lifetime and lower mass compared with systems using expendable cryogens. However, of the six constructed instruments in Fig. 1, all except one (Planck/HFI) use cryogens - our experience in this regard is thus even more limited than for space ultra-low temperature instruments in general.

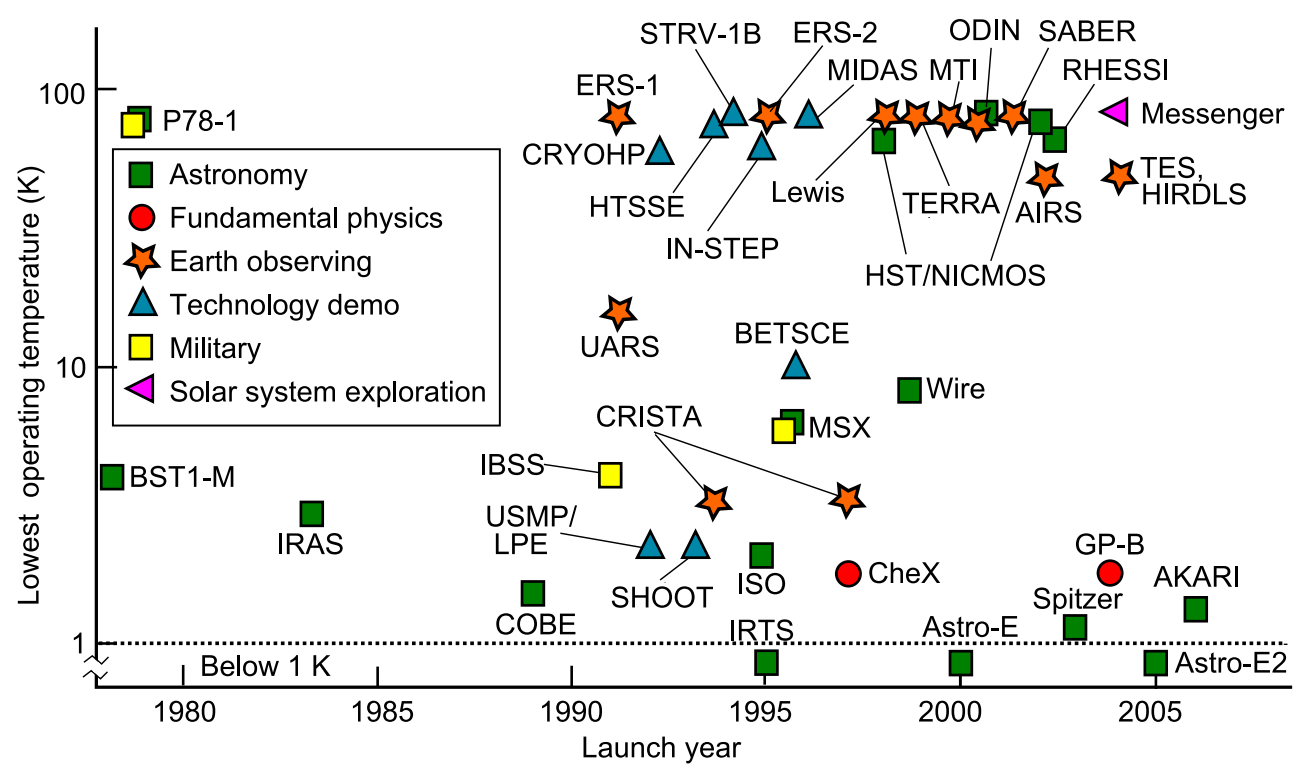

Figure 2. A (no doubt woefully incomplete) list of space cryogenic missions (defined as having a lowest temperature below $100 \mathrm{~K}$ ) launched to date. Missions entirely using passive radiators for cooling are not shown. The information was mostly taken from Refs. 2,3 and 4 . 


\section{SCUBA-2 THERMAL DESIGN}

\subsection{Overview}

The thermal design of the SCUBA-2 instrument has been described in detail elsewhere. ${ }^{6,7}$ A brief summary is given here, followed by more detailed information on some of the critical areas of the design.

The instrument contains two focal planes, each with a mass of approximately $10 \mathrm{~kg}$, and each consisting of four subarrays butted together. The arrays operate from a heat sink at a temperature of about $50 \mathrm{mK}$, separated from the cooling source (the mixing chamber of a dilution fridge) by about 1 metre. The sub-arrays must be individually removable for replacement or repair, and must be supported rigidly (to maintain optical alignment) but with a low heat leak through the supports from the $1-\mathrm{K}$ stage.

The focal planes are contained within a radiation shield maintained at a temperature of about $1 \mathrm{~K}$, called the "1-K box". This is cooled by the still of the dilution fridge, and as with the millikelvin stage, there is a considerable distance between the box and the source of cooling. The $1-\mathrm{K}$ box is surrounded by further radiation shields at $4 \mathrm{~K}$ and $60 \mathrm{~K}$, but these are outside the scope of this paper.

The thermal paths at the 1-K and millikelvin stages are somewhat complex, as can be seen from Fig. 3, which shows the system of thermal links at both temperatures. These consist of flexible copper straps, made from a laminate of many annealed $5 \mathrm{~N}(99.999 \%)$ purity copper foils stacked together to give a high conductance link with sufficient compliance to cope with motion due to thermal contraction. At each end the straps are welded into gold plated electrolytic tough pitch (regular commercial) copper end-pieces, which are bolted to similar pieces to make good thermal contact. Commercial copper is used not only because it is cheaper than bulk $5 \mathrm{~N}$ purity copper, but because it is somewhat harder, and thus less liable to deform after repeated making and breaking of thermal contacts.

The need for the thermal links is two-fold. The cooling power at millikelvin temperatures is extremely limited, and thus the mass at this temperature must also be minimised. It is thus restricted to the detector arrays and their supports, with thermal straps linking them to the mixing chamber of the dilution fridge. The 1-K stage largely consists of the 1-K box. Various points need to be kept below $1.1 \mathrm{~K}$ to keep thermal loads on the millikelvin stage to an acceptable level. In principle, the structure of the box itself could be used to provide cooling to these points: the heat sinks for the wiring to the array, the "warm" end of the array supports, and the driveshaft of the shutter (all shown in Fig. 3).

However, to minimise mass, the box is made from aluminium alloy (6082 T6), which is not a particularly good conductor, especially since at the operating temperature it is likely to be slightly below the superconducting transition temperature. Superconductors make good electrical conductors but poor thermal conductors, though close to the transition temperature the reduction in thermal conductivity is relatively small. In addition, the box is constructed in several sections, bolted together, each joint providing further impedance to heat flow. There was insufficient time in the schedule to discover if the box itself made a good enough conductor. The solution was to use a further system of thermal links providing an all-copper path between the still of the dilution refrigerator and all points on the 1-K box to be cooled. This system, while complex and not exactly light-weight, has functioned extremely well.

\subsection{Specific Areas}

\subsubsection{Material properties}

A problem encountered with SCUBA-2 was the general lack of information on properties of materials at cryogenic temperatures. This problem is of course not unique to SCUBA-2, but the demanding requirements made it a more serious issue than for simpler instruments; few instruments have been built on this scale to operate at such low temperatures. The usual solution to this problem is to stick to the small set of materials with well-known properties, and which are commonly used in cryostats. Unfortunately, we couldn't do this and meet all the requirements of the instrument; this is also likely to be the case for future ULT space instruments. The solution was to choose likely materials and carry out tests during the design process. This is obviously not an ideal situation, since if the materials turn out to have undesirable properties, finding an alternative is likely to cause delay. A programme of tests on materials which look promising for future cryogenic instrumentation, and which is independent of the schedule of any particular instrument, would be extremely useful.

To some extent, tests will probably always be necessary when constructing ULT instruments due to sample to sample variation, which can be extreme even at temperatures as high as $4 \mathrm{~K}$. For example, the low temperature conductivity of pure metals varies by many orders of magnitude depending on the amount and types of impurity. While a range of likely values 


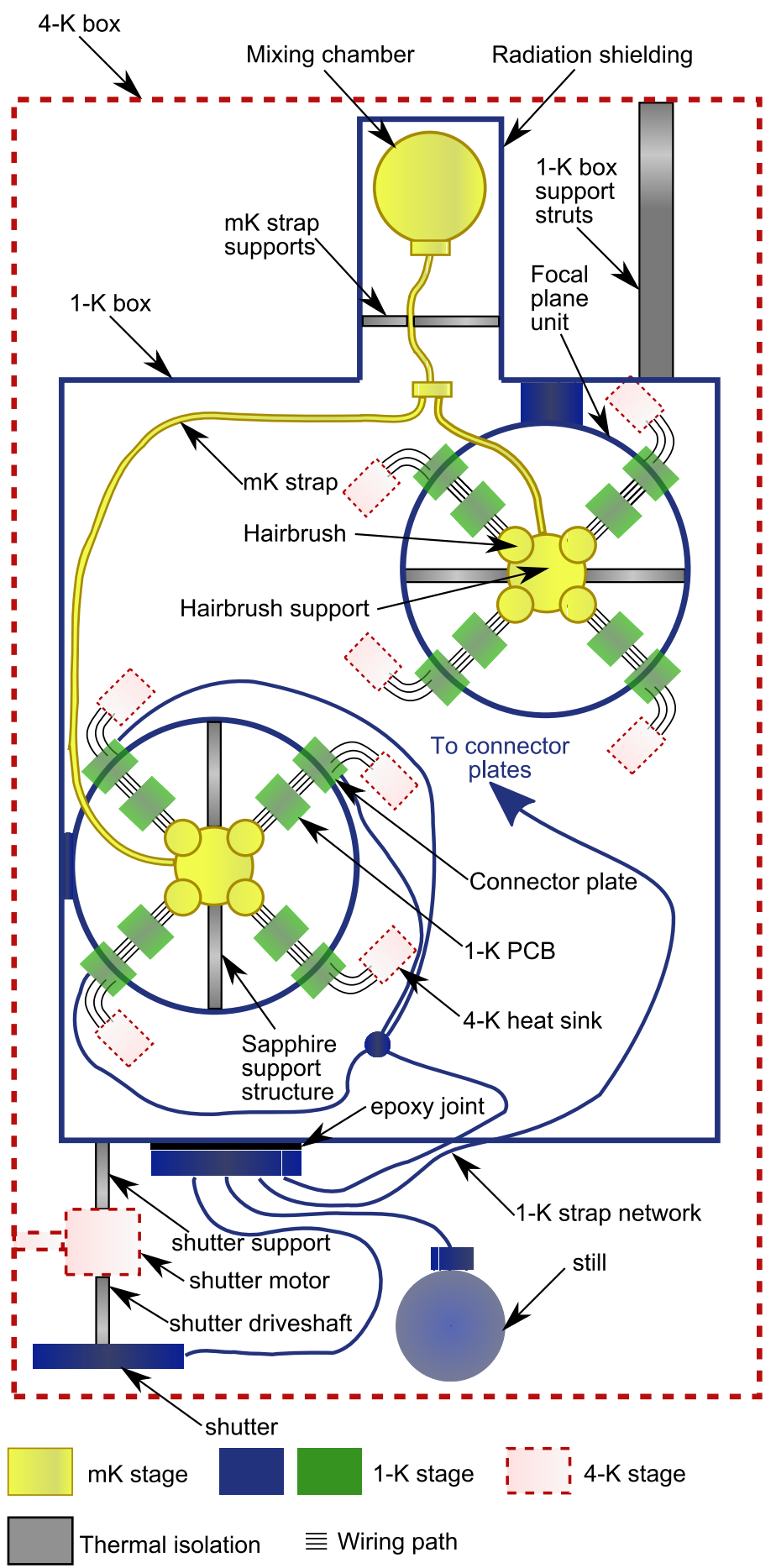

Figure 3. The thermal paths at the 1-K and millikelvin stages in SCUBA-2. To simplify the diagram, the network of $1-\mathrm{K}$ thermal links is only shown in full for one of the two focal plane units. 


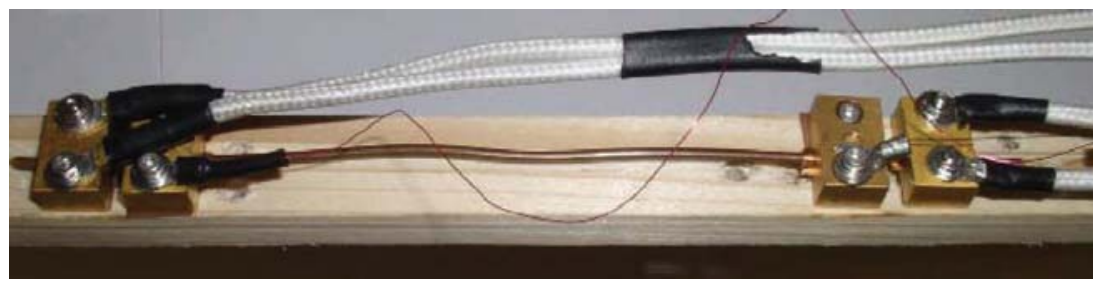

Figure 4. Simple set-up for measuring the electrical resistivity of a metal sample; the thermal conductivity can then be calculated using the Wiedemann-Franz law.

can be predicted if the nominal purity is known, ${ }^{8}$ this range can be wide, particularly for copper. It is often necessary to measure samples of the actual material to be used in thermal straps, with exactly the same heat treatment (annealing) to have confidence in the conductivity.

One technique that can significantly simplify this process is to make use of the Wiedemann-Franz law, ${ }^{9}$ which relates the electrical and thermal conductivity of a metal at low temperatures, and which is generally extremely accurate. ${ }^{8}$ Measuring electrical resistance is a lot easier than thermal conductance, and can be done quite simply by putting a sample on the end of a stick and lowering it (carefully) into a dewar of liquid helium (Fig. 4). For pure metals a rather large current can be required (10 Amps or more); this is conveniently applied with a magnet power supply of the type used to operate an adiabatic demagnetisation refrigerator (ADR).

Sample variation is not only a problem with metals. Other materials can also show significant sample to sample variation. CFRP (carbon reinforced polymer) struts were used to support the 1-K box. To be sure of the conductance, the conductivity of samples from the same lot of material used was measured. Since the Wiedemann-Franz law cannot be used for dielectrics, the thermal conductance was measured directly. Struts made from G-10 (the material normally used for constructing printed circuit boards) were used at higher temperatures. This also carries a risk, since while in practice G-10 appears to have fairly reproducible properties, "G-10" is not in fact a material specification at all, but a specification on the electrical properties of the material.

\subsubsection{Joints}

To make integration possible, a certain number of joints were necessary in the thermal links. Some of these joints were made once during assembly, never to be touched again. However, in order to remove the 1-K box, or to remove individual arrays, some joints have to be broken apart and later remade. Great attention was paid to all of the joints in the straps. The importance of joints is sometimes overlooked, which is unfortunate as the temperature drop across a poorly designed or badly constructed joint can dominate the total temperature difference across a thermal link, and of course it only takes one bad joint to run the performance of an entire thermal link.

Unfortunately, there is a lack of qualitative data on the performance of thermal joints due to the large number of factors involved, such as the bulk and surface properties of the materials being joined, and the quantity and distribution of the force used to join them. Even worse, measurements on the performance at intermediate temperatures between the cryogenic temperature range and room temperature are almost totally lacking, ${ }^{10}$ making modelling the cool-down of an instrument difficult.

However, the general principles involved in making good joints are reasonably well known, ${ }^{11,12}$ and were applied. These consist of maximising contact between the two metallic surfaces by:

- Minimising oxide layers on the surfaces by gold plating (and not using vacuum grease between the surfaces).

- Maximising the force from the bolts holding the joint together; there is good evidence that the nominal area of the joint is unimportant, with joining force being the quantity which determines the conductance. ${ }^{13-15}$ The joints were therefore designed with as many bolts as possible, torqued to as high a force as was reasonable.*

As with bulk metals, electrical resistance measurements can be used to predict the joint conductance via the WiedemannFranz law.

\footnotetext{
* Another point which is sometimes overlooked is that the force obtained by a bolt tightened to a given torque decreases as the bolt diameter increases. The reason to use larger bolt diameters is that they can tolerate higher torques without snapping. However, it is not useful to use larger bolts unless you take advantage of the increase in possible torque.
} 


\subsubsection{1-K box thermal joint}

While copper thermal links were provided between the dilution fridge still and the areas on the 1-K box that had to be cooled to around $1 \mathrm{~K}$, it was still important for the box itself to reach a temperature below $1.5 \mathrm{~K}$ to keep the radiative load to an acceptable level. Therefore good thermal contact was required between the box and the thermal strap running to the still. However, it is notoriously difficult to make good thermal contact to aluminium, due to the oxide layer which builds up almost instantly on clean aluminium.

There are various solutions to this problem. Some good results have been reported with gold plated surfaces. However, it is difficult to gold plate aluminium without ending up with oxide between the gold and the aluminium, and many bad results have also been reported. In any case, carrying out all the cleaning steps required to gold plate a patch on a large aluminium box sufficiently well would be very difficult.

It has been suggested that a large enough force can produce good results with bare aluminium contacts, since (unlike copper), aluminium oxide is softer than the aluminium. However, as with other areas of the design, we did not have time to experiment, but needed a solution with predictable performance.

The answer was to glue a copper pad onto the aluminium box using Stycast 2850 epoxy (this has a thermal contraction which is better matched to metals than unfilled epoxies such as 1260). This, of course, contradicts the rule quoted above that joints should have good metallic contact, since epoxies are thermal insulators. However, the advantage of such a joint is that unlike a pure metal to metal joint, where the inevitable surface roughness means that the two surfaces only actually make contact over a fraction of the nominal surface area, the epoxy flows between the surfaces and makes contact over a much larger area. By keeping the epoxy layer thin, adequate conduction can be achieved. The performance is still poor compared to a good metal to metal joint, but this can be overcome by making the area of the joint large. Unlike a "dry" joint, where total force, not total area, is important, the conductance across an epoxy joint will scale with area.

This approach has worked well for SCUBA-2, and a similar joint was used in the Herschel/SPIRE instrument to replace a bolted copper to aluminium joint to a radiation shield, improving the conductance across the joint dramatically, and allowing the shields to reach the required temperature.

\subsubsection{Sapphire thermally isolating support}

The arrays are supported by the 1-K box. The supports have the conflicting requirements that they must be rigid, in order to maintain the optical alignment of the detectors, yet they must have a sufficiently low thermal conductance that the heat leak from the $1-\mathrm{K}$ box is acceptably small. The obvious way to do this would be to use a rigid material with low thermal conductivity. However, there is insufficient space to achieve this with any known material. The usual solution to this problem (particularly for space instruments) is to use Kevlar ${ }^{\circledR}$ thread. ${ }^{16-18}$ However, there are various disadvantages to Kevlar. Since it expands upon cooling, and also creeps with time, it is necessary to keep it under tension. Also, extreme care is required to keep the surfaces that it passes over smooth, as rough surfaces can cause the thread to snap, leading to a catastrophic failure.

We therefore adopted a completely different approach, using joints which take advantage of the poor thermal conductance across pairs of sapphire surfaces. As described above, metal surfaces are always rough at the microscopic level, and will only touch at the highest points. As force is applied across the joint, the contact points will deform, increasing the total contact area, giving a conductance that increases with force. Sapphire is much harder than a metal, and therefore deforms much less under pressure, keeping a low true contact area. Powder placed between the surfaces gives a further decrease in conductance. A test campaign ${ }^{11}$ was carried out different designs of joint, varying the clamping force, joint area, and presence and type of powder. The measurements were sped up by showing that the performance at millikelvin temperatures was correlated with that at a temperature of $4 \mathrm{~K}$, and then carrying out most measurements at the much more easily attained temperature of $4 \mathrm{~K}$.

A single joint of the best design resulting from the test programme is shown in Fig. 5, with a cotton bud for scale. Each joint contains two pairs of sapphire surfaces, so that pressure can be applied across the sapphire pairs without the screws thermally shorting the joint. An individual joint is free to rotate in two degrees of freedom (in practice, the hot and cold sides of the joint do rotate against each other but are remarkably resistant to being pulled apart). Therefore, the full support (Fig. 6) is constructed from four joints set at an angle of 45 degrees to each other to provide a support with no degrees of freedom. 

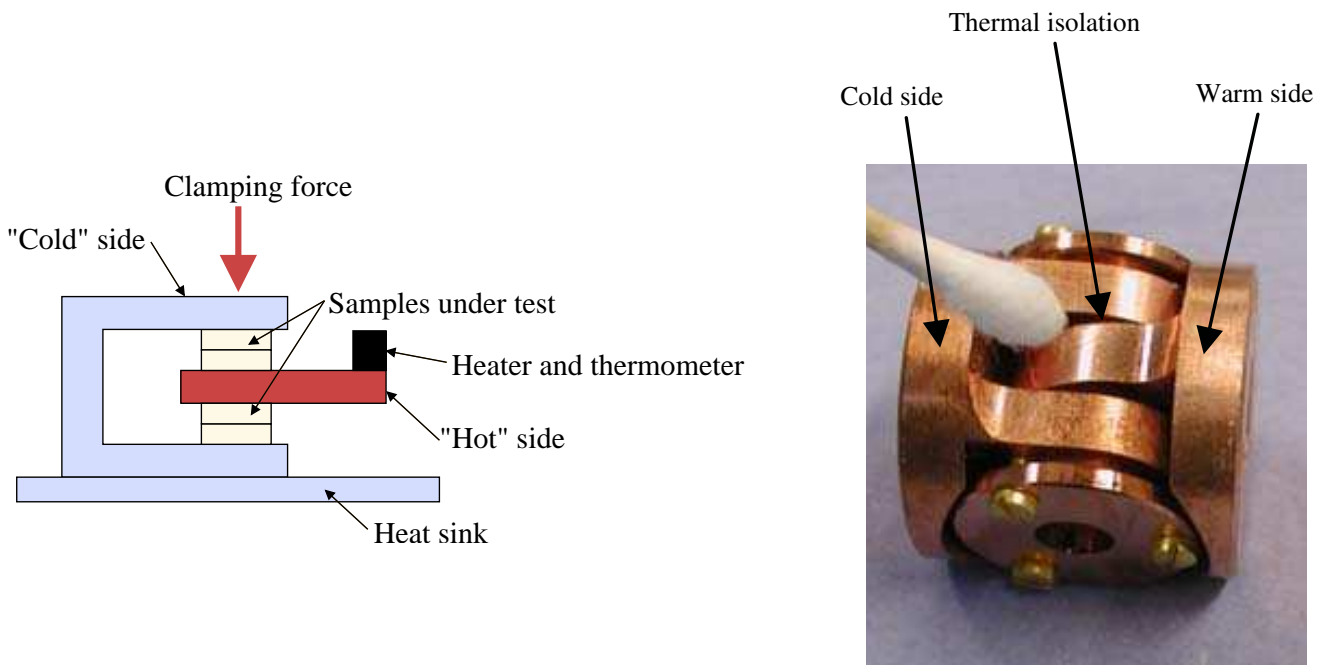

Figure 5. Schematic and photograph of a single sapphire thermal isolation joint.

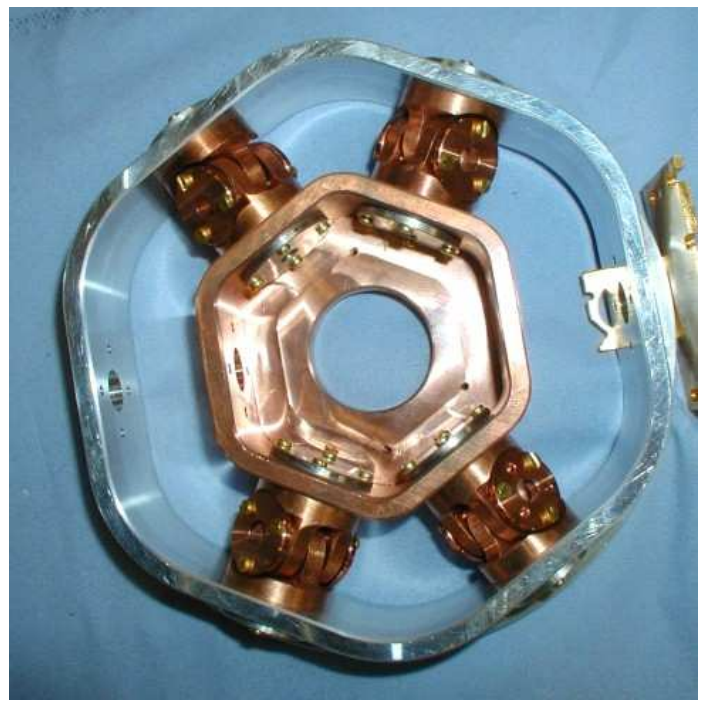

Figure 6. A prototype thermal isolation structure.

Tests on a prototype full support showed a heat leak of just over $2.5 \mu \mathrm{W}$ from $80 \mathrm{mK}$ to $1.1 \mathrm{~K}$, within the requirements for the instrument. The mechanical requirements were also met; repeated loading of the support up to $10 \mathrm{~kg}$ showed no hysteresis in the measured deflection, and a load of $5 \mathrm{~kg}$ produced a repeatable deflection of $8 \mu \mathrm{m}$. The support was left for 65 hours under a load of $5 \mathrm{~kg}$, with no further movement or creep seen.

\subsubsection{The hairbrush}

The detector arrays in SCUBA-2 are considerably larger than anything which has been constructed before for sub-millimetre astronomy, and the size of the arrays creates a large problem for the thermal design. The heat generated by the operation of the detectors must be removed efficiently or they will overheat and cease to operate. The thin silicon wafers do not have sufficiently good conductance to be able to carry the heat out to the edges; thermal contact is required across the entire back of the array.

However, due to the mismatch in thermal contraction between silicon and materials which are useful as heat sinks, mounting of arrays must be done very carefully, and even carefully mounted infra-red photodetector arrays have been 


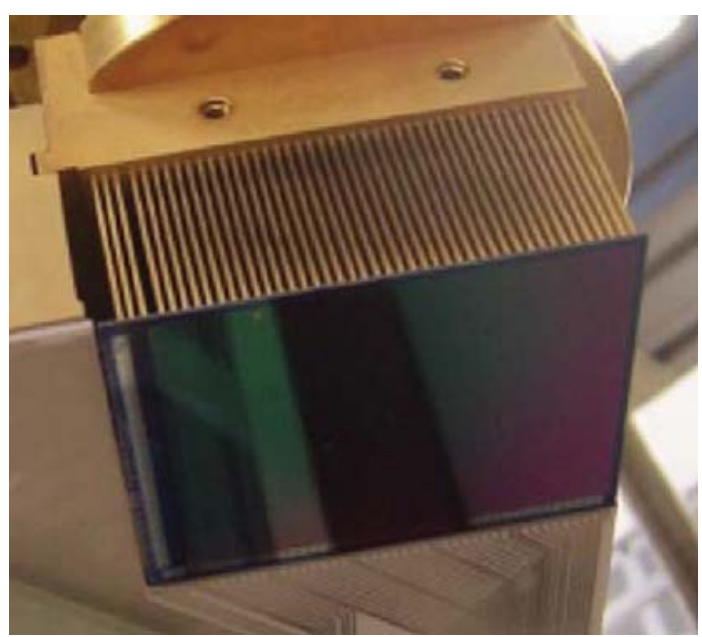

Figure 7. A SCUBA-2 sub-array bonded to the beryllium copper "hairbrush" heat sink.

known to break when cooled. Gluing a SCUBA-2 array down onto a chunk of copper, while an excellent solution from a thermal point of view, would not be desirable. The solution to this problem is a device known as the "hairbrush", and shown in Fig. 7. This consists of a piece of a high conductivity beryllium copper alloy, which has been cut into a forest of individual tines by electrical discharge machining (EDM). ${ }^{\dagger}$ Each pixel has its own leg, bonded to the silicon wafer using Stycast 1260 epoxy. During cool-down, the thin legs can move as the silicon contracts, taking up the relative thermal contraction between the silicon and the base of the hairbrush. Beryllium copper is a very springy material, and therefore has suitable mechanical properties, while having reasonably good thermal conductivity.

However, having machined a hairbrush, the problem does not end there. It is important that the glue doesn't bridge the tines, otherwise they will not be able to move independently as necessary. Once an array has been glued down, it is practically impossible to remove it again, and even harder to examine the joints in order to find out whether you would want to. By the time an array has reached the stage of being glued to the hairbrush, it has been through an extensive (and expensive) series of fabrication steps on two continents, and destroying one at this stage would not be pleasant.

The solution (after another lengthy test programme) was to apply drops of glue individually onto each of the 1000+ tines on a hairbrush, before carefully lowering the wafer into place using a jig. This process has to be completed reasonably quickly, before the epoxy has had time to set. This is done by using a desktop robot ${ }^{\ddagger}$. Connected to a container of epoxy, it can be programmed to deliver a metered quantity of epoxy onto each tine, rapidly covering the whole array.

\section{LESSONS LEARNED}

As well as the specific issues involved in the SCUBA-2 design, described above, various more general lessons have emerged from the design, construction and testing of SCUBA-2.

\subsection{Positive}

The thermal design was highly successful, with the instrument cooling down to below the required temperature, faster than required, on the very first cooldown. I believe that various aspects of the design process have contributed to this success:

- A large amount of effort went into the thermal design, which was treated as an integral part of the overall instrument design, rather than letting the mechanical requirements dominate as is sometimes allowed to happen.

\footnotetext{
${ }^{\dagger}$ Although not visible in Fig. 7, the ends of the tines splay out to increase the contact area above the cross-section of the legs, to improve conduction across the glue join. Increasing the leg cross-section would have reduced flexibility too much. Fortunately, it is actually possible to machine such a structure using EDM.

${ }^{\ddagger}$ Janome, 1822 Brummel Ave., Elk Grove Village, IL 60007, USA
} 
- The project team already had experience with instruments in this temperature range. There can be a large difference between the design of a instrument operating at $2 \mathrm{~K}$ and $100 \mathrm{mK}$, and experience at higher temperatures can be misleading. § $^{-1}$

- We took advantage of cryogenic experience outside astronomy on review boards, to give extra perspective.

- A detailed thermal model was created and updated in parallel with changes to the mechanical design.

- The thermal design was treated as a single system, rather than a series of sub-systems linked only by well defined interfaces.

- The thermal performance of subsystems was tested, where possible, before finalising the design. Where we weren't able to this, we built in large safety margins instead.

I believe that these points are important, but — even for space instruments — they are not all necessarily followed. The thermal and mechanical design of an instrument is intimately related, and a failure to fully consider thermal aspects of a design at an early enough stage can lead to less than optimal design. " Likewise, while splitting the overall design into independent subsystems is the obvious choice for a complex instrument, it can lead to a poor final design from a thermal point of view.

For example, an instrument may have a maximum tolerable temperature drop across a thermal path. If the path includes more than one sub-system, we must divide the total temperature drop into a contribution from each subsystem. The temperature drop depends on the power passing along the link, which is probably largely generated by the subsystem at one end, with other subsystems adding some power due to thermal conduction along supports from a higher temperature stage. Therefore we must also set power budgets for each stage. However, the requirements for each sub-system must be set before the detailed design takes place, otherwise we don't know what we are trying to design each sub-system to do. Therefore any decision on the requirements for each sub-system must to some extent be a guess, with the risk that subsystems are given unreasonably tight requirements, or have requirements which are looser than necessary, making other sub-systems harder to construct. The result is to turn a relatively simple system into a much more complex one; ultimately, all we care about is the total temperature drop across the link.

In practice, with a complex instrument designed by multi-institutional teams, division into sub-systems is probably inevitable. However, the resulting problems can be minimised by very careful choice of what constitutes a sub-system, rather than basing it entirely on obvious mechanical considerations, and by a strong oversight of the overall thermal design along with a willingness to adjust sub-system requirements where appropriate.

An example of the need to define sub-systems very carefully occurs in the case of a demountable joint in a thermal link which joins two sub-systems. The obvious choice from a mechanical point of view is to take the interface between sub-systems at the joint in the thermal link. However, from a thermal point of view this is a very poor choice. A joint is a subsystem in itself, which should be designed and qualified separately. Having the two sides of the joint the responsibility of different groups or organisations is not likely to lead to good performance (and whose thermal budget does the temperature drop across the interface itself come out of?). This situation has happened, and in a space instrument too.

\subsection{Negative}

There are also various aspects of the design process that I would not advocate repeating:

- After the main design was completed, to some extent we lost our view of the overall picture. At this point, the mechanical design was allowed to dominate. While each change to the mechanical design was verified as being acceptable against the thermal model, the thermal performance was degraded in a series of small steps, without stepping back to look at the overall picture to see if a more radical redesign might improve margins and lead to a simpler overall design.

- As mentioned above, it wasn't possible to test all subsystems, relying instead on large safety margins. This probably led to a somewhat overengineered system. For example, if we had time to conduct tests on the conductance through the $1-\mathrm{K}$ box, we might have been able to eliminate some of the copper thermal links around the box, reducing the mass. Overengineering is unlikely to be an option for future ULT space missions.

\footnotetext{
${ }^{\S}$ To give an example, sapphire is useful for good thermal conduction and electrical isolation at $2 \mathrm{~K}$, but not at $100 \mathrm{mK}$ (the conductivity of a dielectric is proportional to $T^{3}$, where $T$ is temperature, and so the conductivity falls by a factor of 8000 between $2 \mathrm{~K}$ and $100 \mathrm{mK}$ ). Another example is that pure aluminium is a very good thermal conductor at $2 \mathrm{~K}$, but not at 100 or $300 \mathrm{mK}$ where it becomes superconducting.

"Electrical design can also have a large impact on the difficulty of the thermal design. For example, electrical isolation is relatively easy to achieve in a thermal link near room temperature, but very difficult at $300 \mathrm{mK}$.
} 


\section{CONCLUSIONS}

There are currently various planned astronomy space missions that will require ultra low temperature (ULT) operation (temperatures below $1 \mathrm{~K}$ ). While there is now a considerable body of experience in the design of such instruments, actual heritage of successful instruments in flight is extremely limited. Moreover, the next generation of instruments are likely to have considerably more demanding requirements than for existing ones.

In designing such instruments, we should therefore take advantage of experience with ground based instruments (both within and outside astronomy) at these temperatures. One such instrument is SCUBA-2, probably the largest and (thermally) most complex astronomical instrument ever built to operate at ultra-low temperatures. Despite this complexity, the thermal design was highly successful. Various components were developed specifically in order to meet the requirements of SCUBA-2, such as the "hairbrush" and sapphire thermally isolating support, described here in some detail.

More generally, various principles were adopted which contributed to the successful design. To summarise these, the thermal design was considered to be an integral part of the overall design from the beginning, and was allowed to dictate mechanical design where necessary. The thermal design was considered at the level of the overall system, rather than arbitrarily defining requirements for each sub-system. Where possible, the thermal performance of each sub-system was verified. Where this wasn't possible, large safety margins were applied. A detailed thermal model of the entire system was maintained, and any change to the mechanical design only took place after the model had been used to verify that the resulting changes to the thermal design still maintained the specifications.

There will be many interesting challenges ahead in the next few years in the design of ULT instrumentation for space, and there is much hard work to be done. We will make life easier for ourselves by taking heed of experience (good and bad) not only with previous space missions, but also from ground based instruments such as SCUBA-2.

\section{REFERENCES}

[1] Triqueneaux, S. et al., "Optimised (low temperature) cooling chains for IR and X-ray missions." Presentation at the 3rd European Space Cryogenics Workshop, ESA/ESTEC, November 2007, ESA CD reference number WPP-277, ISSN 1922-6656.

[2] Collaudin, B. and Rando, N., "Cryogenics in space: a review of the missions and of the technologies," Cryogenics 40, 797-819 (2000).

[3] Seigel, P. H., “THz instruments for space," IEEE Trans. Antennas and Propagation 55(11), 2957-2965 (2007).

[4] Ross Jr., R. G. and Boyle, R. F., "An overview of NASA space cryocooler programs - 2006," in [Proceedings of the International Cryocooler Conference, Annapolis, MD. ], (2006).

[5] Holland, W., MacIntosh, M., Fairley, A., Kelly, D., Montgomery, D., Gostick, D., Atad-Ettedgui, E., Ellis, M., Robson, I., Hollister, M., Woodcraft, A., Ade, P., Walker, I., Irwin, K., Hilton, G., Duncan, W., Reintsema, C., Walton, A., Parkes, W., Dunare, C., Fich, M., Kycia, J., Halpern, M., Scott, D., Gibb, A., Molnar, J., Chapin, E., Bintley, D., Craig, S., Chylek, T., Jenness, T., Economou, F., and Davis, G., "SCUBA-2: a 10,000 pixel submillimeter camera for the James Clerk Maxwell Telescope," Proc. SPIE 6275, 62751E-1-15 (2006).

[6] Woodcraft, A. L., Gannaway, F. C., Gostick, D. C., and Bintley, D., "Thermal design of the SCUBA-2 instrument detector stage and enclosure," Proc. SPIE 5498, 446-454 (2004).

[7] Gostick, D., Montgomery, D., Wall, B., McGregor, H., Cliffe, M., Woodcraft, A., and Gannaway, F., "The cryomechanical design of SCUBA-2: a wide-field imager for the James Clerk Maxwell Telescope," Proc. SPIE 5492, 1743-1754 (2004).

[8] Woodcraft, A. L., "Recommended values for the thermal conductivity of aluminium of different purities in the cryogenic to room temperature range, and a comparison with copper," Cryogenics 45(9), 626-636 (2005).

[9] Pobell, F., [Matter and Methods at Low Temperatures], Springer (1992).

[10] Gmelin, E., Asen-Palmer, M., Reuther, M., and Villar, R., "Thermal boundary resistance of mechanical contacts between solids at sub-ambient temperatures," J. Phys. D-App. Phys. 32(6), R19-R43 (1999).

[11] Bintley, D., Woodcraft, A. L., and Gannaway, F. C., "Millikelvin thermal conductance measurements of compact rigid thermal isolation joints using sapphire-sapphire contacts, and of copper and beryllium-copper demountable thermal contacts," Cryogenics 47(5-6), 333-342 (2007).

[12] Didschuns, I., Woodcraft, A. L., Bintley, D., and Hargrave, P. C., "Thermal conductance measurements of bolted copper to copper joints at sub-Kelvin temperatures," Cryogenics 44(5), 293-299 (2004). 
[13] Berman, R., "Some experiments on thermal contact at low temperatures," J. App. Phys. 27(4), 318-323 (1956).

[14] Berman, R. and Mate, C. F., "Thermal contact at low temperatures," Nature 182(4650), 1661-1663 (1958).

[15] Okamoto, T., Fukuyama, H., Ishimoto, H., and Ogawa, S., "Electrical resistance of screw-fastened thermal joints for ultra-low temperatures," Rev. Sci. Instrum. 61(4), 1332-1334 (1990).

[16] Roach, P. R., "Kevlar support for thermal isolation at low temperatures," Review of Scientific Instruments 63(5), 3216-17 (1992).

[17] Duband, L., Hui, L., and Lange, A., "Thermal isolation of large loads at low temperature using Kevlar rope," Cryogenics 33(6), 643-647 (1993).

[18] Freund, M. M., Duband, L., Lange, A. E., Matsumoto, T., Murakami, H., Hirao, T., and Sato, S., "Design and flight performance of a space borne ${ }^{3} \mathrm{He}$ refrigerator for the infrared telescope in space," Cryogenics 38(4), 435-443 (1998). 